\title{
Small RNAs generated by bidirectional transcription mediate silencing of RXLR effector genes in the oomycete Phytophthora sojae
}

Qinhu Wang ${ }^{1}$, Tingting $\mathrm{Li}^{2}$, Chengcheng Zhong ${ }^{1}$, Shizhi Luo ${ }^{1}, \mathrm{Ke} X \mathrm{U}^{1}$, Biao Gu${ }^{1}$, Yuling Meng², Brett M. Tyler ${ }^{3}$ and Weixing $\operatorname{Shan}^{2^{*}}$ (D)

\begin{abstract}
Oomycete pathogens secrete hundreds of effectors, including avirulence proteins that trigger host genotypespecific resistance response, to manipulate host immunity and facilitate infection. Sequence and expression variations of avirulence genes in pathogens are well known to be responsible for loss of host genotype-specific disease resistance. However, little is known on the underlying mechanisms associated with virulence variation in the diploid Phytophthora pathogens. We report in this study that the endogenous small RNAs (sRNAs) are involved in the variation of expression of avirulence gene Avr1b in P. sojae. The sRNAs were originated from the natural antisense transcripts of Avr1b. We further showed that the sense and antisense expression of Avrib were programmed by the 10-base deletions in their promoter regions. Expanded analysis showed that up to 31\% of the P. sojae RXLR effector genes were associated with sRNAs. Genome analysis further showed that the 9-bp and 10-bp insertion/deletion variants were significantly enriched in the regulatory regions of RXLR effector genes. These results indicate that the expression of RXLR effector genes are programmed by significantly enriched variations in their regulatory regions that lead to the variations in bidirectional transcription, which likely further affect production of endogenous sRNAs and silencing of homologous RXLR effector genes of Phytophthora pathogens.
\end{abstract}

Keywords: Oomycete, Phytophthora, Avr1b, Small RNA, RXLR effectors, Antisense transcripts, INDEL variation

\section{Background}

Oomycetes are eukaryotic pathogens causing severe damages to crops (Kamoun et al. 2015). For example, the worldwide annual losses caused by the Irish potato famine pathogen Phytophthora infestans is about $\$ 6.7$ billion (Haverkort et al. 2008; Haas et al. 2009). The losses caused by soybean root and stem rot pathogen Phytophthora sojae is estimated at $\$ 1-2$ billion per year (Wrather and Koenning 2006; Tyler 2007). Oomycete pathogens secrete a large array of effector proteins to manipulate host immunity and facilitate infection (Kamoun 2006; Stassen and Van den Ackerveken 2011). Many effectors, such as the RXLR family effectors (Jiang et al. 2008; Haas et al.

\footnotetext{
* Correspondence: wxshan@nwafu.edu.cn

${ }^{2}$ State Key Laboratory of Crop Stress Biology for Arid Areas, and College of Agronomy, Northwest A\&F University, Yangling 712100, Shaanxi, China Full list of author information is available at the end of the article
}

2009), are able to trigger or suppress programmed cell death in planta (Oh et al. 2009; Wang et al. 2011). If an effector is recognized by a specialized host resistance (R) protein, typically a nucleotide-binding site and leucine-rich repeat protein, it triggers hypersensitive response (HR) and prevents proliferation of the pathogen, and is called avirulence (Avr) protein (Jones and Dangl 2006). Interestingly, nearly all the avirulence genes identified in oomycetes encode RXLR effectors (Jiang and Tyler 2012; Anderson et al. 2015).

The successful pathogens have evolved diverse genetic strategies to evade the recognition by host plants. A variety of genetic variations in Avr genes, such as nucleotide substitution, insertion, deletion, frameshift, stop-codon mutation, truncation and copy number variation, are shown involved in the generation of novel types of virulent strains (Shan et al. 2004; Tyler et al. 
2006; Qutob et al. 2009). Reversible virulence/avirulence variations of Phytophthora pathogens were frequently observed during asexual reproduction (Rutherford et al. 1985; Malvick and Percich 1998; Abu-El Samen et al. 2003). One possible reason is the suppressed expression of avirulence genes, thus leading to loss of race-specific resistance. $P$. sojae avirulence gene Avr1b can trigger HR in soybean plants carrying resistance gene Rps1b (Shan et al. 2004). Interestingly, epigenetic variation is also observed in the regulation of Avrlb phenotype in P. sojae. Two P. sojae strains, P6594 and P6497, carry the identical Avr1b gene, but displayed distinct phenotypes on Rps $1 b$ soybean plants. The accumulation of $A v r 1 b$ mRNA in strains P6594 and P6497 are closely related to their $A v r 1 b^{+}$and $A v r 1 b^{-}$phenotypes, respectively. $A v r 1 b$ of $P$. sojae strain P7064 $\left(A v r 1 b^{+}\right)$has a synonymous single nucleotide polymorphism and is an epiallele of $A v r 1 b^{\mathrm{P} 6497}$ in fact. Genetic cross of strain P6497 and strain P7064 defined that the expression of $P$. sojae Avrlb is regulated by a trans-acting factor, and these two genes determine the Avrlb phenotype (Shan et al. 2004). However, how the mRNA level of $A v r 1 b$ is regulated remains unclear.

Two popular hypotheses could address the epigenetic phenomenon at Avr1b locus: one is DNA methylation leading to transcriptional gene silencing, and the other is RNA silencing at posttranscriptional level and guided by small RNA (sRNA). However, it is known in oomycetes that transgene-triggered transcriptional gene silencing is not associated with DNA methylation (van West et al. 1999; Judelson and Tani 2007; van West et al. 2008). Two important gene silencing components involved in DNA methylation, cytosine methyltransferases and RNA polymerase IV, are also absent in the $P$. infestans genome (Vetukuri et al. 2011a). Moreover, many sRNAs are discovered in oomycetes and some of them were shown to be associated with silencing of RXLR effector genes (Vetukuri et al. 2012; Fahlgren et al. 2013; Qutob et al. 2013; Jia et al. 2017).

In this paper, we show that the silencing of Avrlb in $P$. sojae is associated with sRNA derived from its natural antisense transcripts (NATs). Close examination showed that the bidirectional transcription of Avr1b locus is likely programmed by the insertion/deletion (INDEL) variations in its regulatory regions. Genome analysis further showed that the 9-bp and 10-bp INDEL variants were significantly enriched in the regulatory regions of RXLR effector genes. Finally, analysis of the gene expression showed that up to 31\% of RXLR effector genes in $P$. sojae were associated with sRNAs. These results indicated the expression of RXLR effector genes is likely programmed by the significantly enriched variations in their regulatory regions, leading to the variations in bidirectional transcription, production of endogenous
sRNAs, and efficient silencing of homologous RXLR effector genes of Phytophthora pathogens.

\section{Results}

\section{Identification of $A v r 1 b$-associated sRNAs}

$A v r 1 b$ of $P$. sojae is the first avirulence gene cloned from oomycete pathogens and is recognized by soybeans carrying Rps1b (Shan et al. 2004). Two types of virulent P. sojae strains on Rps1b soybeans were identified, with one represented by P7076 that carries Avr1b allele significantly changed in the coding sequence, and the second represented by $\mathrm{P} 6497$ that carries the intact coding sequence but deficient with $A v r 1 b$ transcripts. Accumulating evidence indicate that sRNA -mediated post-transcriptional gene silencing is important for the epigenetic variation in Phytophthora. Therefore, to examine underlying mechanisms in the diploid P. sojae for the loss of Avr1b transcript accumulation in the virulent P6497, we performed sRNA deep sequencing and investigated the sRNA reads homologous to Avr1b. The results showed that there were two classes of sRNAs homologous to Avrlb (Fig. 1a, b), with one species being peaked at $25 \mathrm{nt}$ (Fig. 1c) and designated as Avrlb-siRNAs. The second class was the antisense sRNA that spans the two neighboring Avrlb-siRNAs, which was named Avrlb-asRNA hereafter (Fig. 1a, b). Taken together, we infer that the silencing of $A v r 1 b$ in the virulent strain P6497 may be related to these $A v r 1 b$-associated sRNA.

\section{Avr $1 b$-associated sRNAs accumulate in virulent strains}

To examine whether the deep sequencing identified sRNAs were associated with the regulation of $A v r 1 b$ phenotype, soybean plants (rps $1 b)$ infected with $P$. sojae strains P6497, P7064, and P7076 were prepared and used for Northern analysis, respectively. The results showed that the Avr1b-siRNAs were specifically accumulated in the virulent strains P6497 and P7076, but not in the avirulent strain P7064 (Fig. 2a). By using the probe designed for Avr $1 b$-asRNA, two different sizes of sRNAs were detected (Fig. 2b), with one being the Avr1b-asRNA but longer (about $40 \mathrm{nt}$ ) than the deep sequencing reads which ranged from 18 to $30 \mathrm{nt}$ (Fig. 2b). The other one is a soybean sRNA (designated as Gma-1b-asRNA) that found in all the infection samples assayed (Fig. 2b). Avrlb-asRNA was also specifically accumulated in the virulent strains P6497 and P7076, but not in the avirulent strain P7064 (Fig. 2b). In addition, we found that Avrlb-asRNA is single-stranded, since its antisense strand was not detectable (Fig. 2c). Therefore, both Avr $1 b$-siRNA and $A v r 1 b$-asRNA accumulation were coincided with the virulent $A v r 1 b^{-}$phenotype.

In further, we noticed that Avrlb-asRNA was accumulated earlier than Avrlb-siRNAs during the infection (Fig. 2a, b), suggesting that Avr1b-asRNA might play as the 

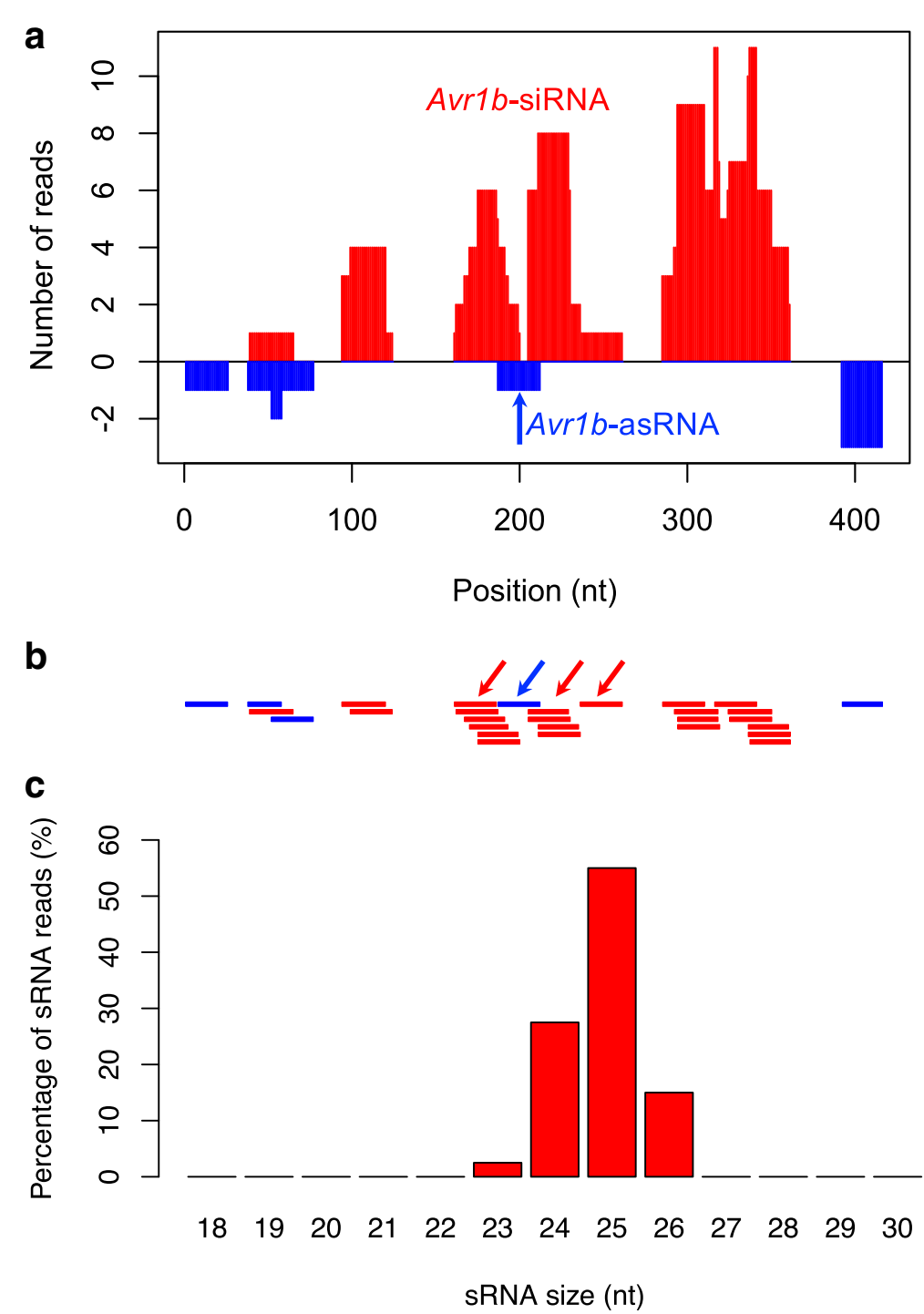

Fig. 1 Identification of P. sojae Avrlb sRNAs. a The abundance distribution of Avrlb sRNA reads homologous to the ORF region. The sense sRNA reads (above the $x$-axis) are highlighted in red, and the antisense sRNA reads (below the $x$-axis) are highlighted in blue. $\mathbf{b}$ The position distribution of Avr1b reads homologous to the ORF region. The red arrows indicate Avrib-siRNA molecules, and the blue arrow that spans two red reads is Avrib-asRNA. The sRNA reads and reference gene sequences are all from P. sojae strain P6497. The position and length of sRNA reads are drawn in scale. $\mathbf{c}$ The size distribution of Avrib-siRNAs

trigger of $A v r 1 b$ silencing. The detection of siRNAs homologous to $A v r 1 b$ indicated that $A v r 1 b$ was transcribed but silenced in the virulent strains P6497 and P7076. Thus, this species of siRNAs was likely the posttranscriptional silencing signal of $A v r 1 b$ in P6497 and P7076. Compared to the soybean sRNA Gma-1$b$-asRNA that was instantly detected during soybean infection (or U6 in Fig. 2e), the intensity of $A v r 1 b$ siRNAs in P6497 was much stronger than that in P7076 (Fig. 2b). This probably explains why the transcripts of virulent $A v r 1 b$ allele was detected easily in P7076 (Shan et al. 2004).
Expression of multiple avirulence genes in $P$. sojae lacks homologous sRNA

Avr1k is an avirulence gene in P. sojae strains P6497 and P7064 but virulent in strain P7076 (Song et al. 2013). We hence performed Northern hybridization to verify if sRNAs were associated with the virulent allele of Avr1k in P7076. Consistently, we found that while the homologs sRNAs were detected for the virulent $A v r 1 k^{\mathrm{P} 7076}$, no homologous sRNA accumulation was detected for the avirulent strains carrying $A v r 1 k^{\mathrm{P} 6497}$ and $A v r 1 k^{\mathrm{P} 7064}$, respectively (Fig. 2d). Therefore, it seems that the expression of both $A v r 1 b$ 


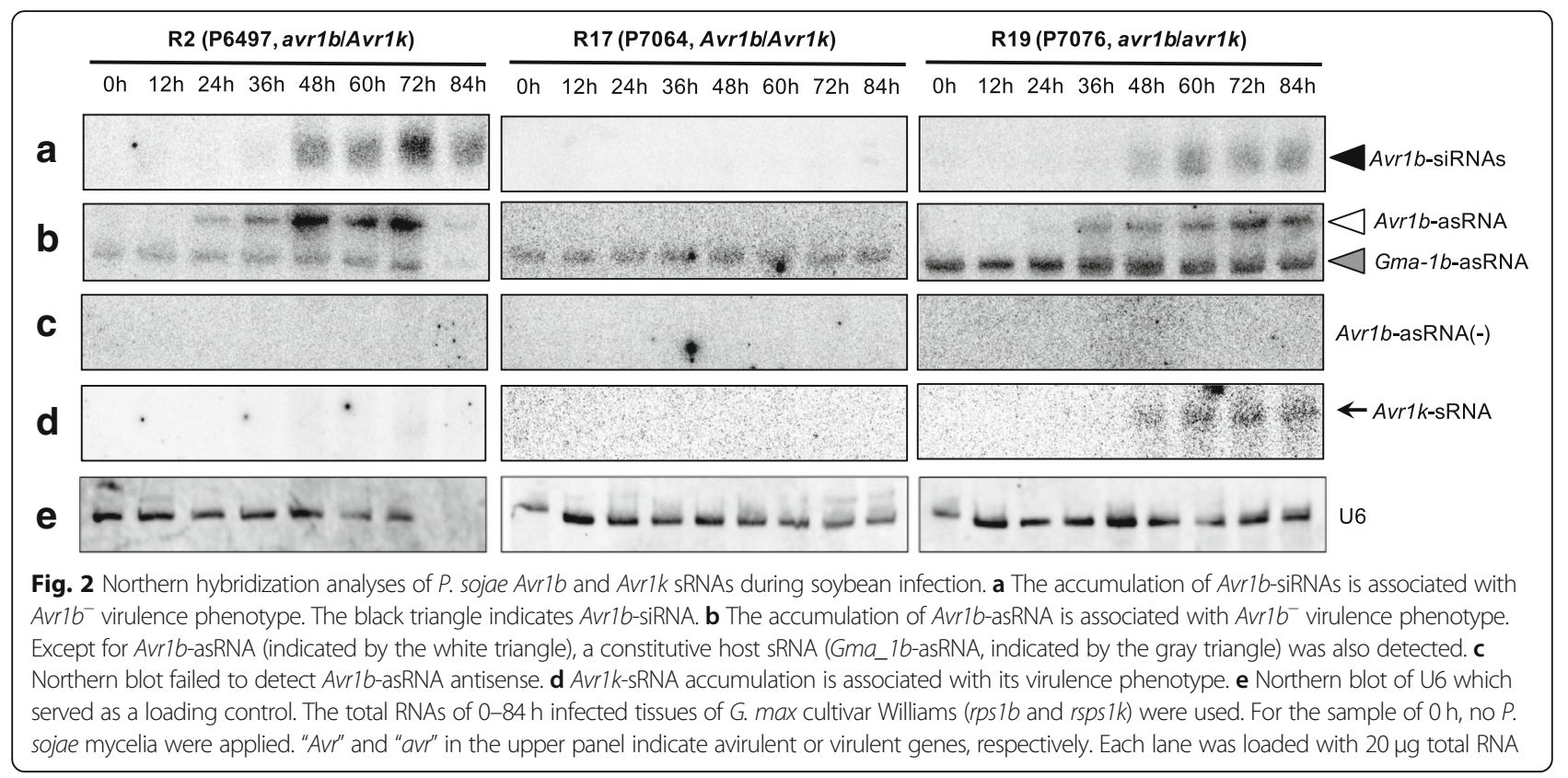

and Avr1k is associated with the absence of sRNAs homologous to the avirulence gene. Inspired by these phenomena, we further examined the presence and absence of siRNA homologous to more avirulence genes. P. sojae strain P6497 carries seven cloned Avr genes. While $A v r 1 b^{\mathrm{P} 6497}$ is a virulent allele, all the rest six $A v r^{\mathrm{P} 6497}$ genes are avirulent (Fig. 3). The results showed that five of the six Avr genes in P6497, Avr3a/5 $5^{\mathrm{P} 6497}, A v r 3 b^{\mathrm{P} 6497}, A v r 3 c^{\mathrm{P} 6497}$, Avr4/ $6^{\mathrm{P} 6497}$, and $A v r 1 k^{\mathrm{P} 6497}$, except $A v r 1 a^{\mathrm{P} 6497}$, had no siRNAs homologous to their open reading frames (ORFs) (Fig. 3). These results suggest that sRNAs are very likely involved in the regulation of the RXLR effector gene expressions in P. sojae.

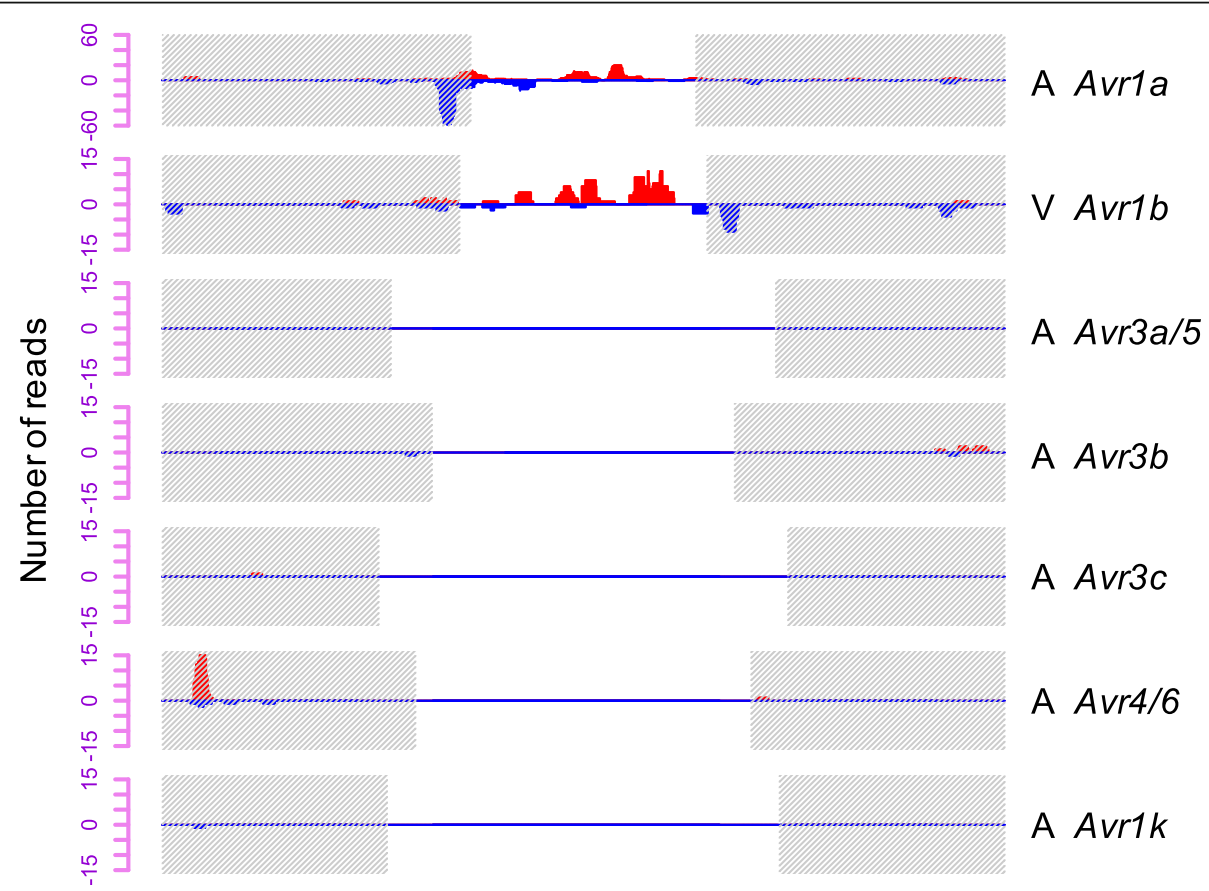

Fig. 3 The abundance distribution of sRNA reads homologous to the functional Avr genes in P. sojae strain P6497. The sense sRNA reads (above the x-axis) are shown in red, and the antisense sRNA reads (below the $x$-axis) are shown in blue. The shaded regions indicate the 5' or 3' 500 bp flanking sequences 


\section{Bidirectional transcription mediates the production of Avr1b-asRNA}

To investigate where the $A v r 1 b$-asRNA was originated from, we performed a sequence similarity search in the P. sojae genome. The result indicated that $A v r 1 b$-asRNA was derived from $A v r 1 b$ or $A v h 1 b$ antisense strand in the virulent strain P6497. However, in strain P7076 which lacks $A v h 1 b$ (Shan et al. 2004), $A v r 1 b$-asRNA was also accumulated (Fig. 2b), indicating that the $A v r 1 b$-asRNA was derived from the antisense strand of $A v r 1 b$.

Taking together of the results above, the $A v r 1 b$ region is obviously transcribed in a bidirectional manner. Sequence analysis showed that the $A v r 1 b$ sense strand encodes Avr1b elicitor, while its antisense strand is predicted to encode an unknown protein with 100 amino acid residues (Fig. 4a). Hence, the dsRNA, predicted to be formed by natural sense and antisense transcripts (NATs) of $A v r 1 b$ locus, is likely the precursor of $A v r 1 b$-asRNA. Avrl$b$-asRNA is located in the $3^{\prime}$ untranslated regions (UTR) of antisense $A v r 1 b$. We infer that $A v r 1 b$-asRNA is derived from the 3'-UTR part of antisense $A v r 1 b$ in the NATs formed by $A v r 1 b(+)$ and $A v r 1 b(-)$.

By using strand-specific RT-PCR assay (Fig. 4a), we further verified the bidirectional transcription of $A v r 1 b$ locus in P. sojae strains P6497, P7064, and P7076. We found that both the sense and antisense strands of $A v r 1 b$ were transcribed (Fig. 4b). Consistently, the Avr1b-asRNA-deriving $A v r 1 b(-)$ was transcribed in virulent strains $\mathrm{P} 6497$ and P7076 during infection, while no $A v r 1 b(-)$ transcripts were detected in the avirulent strain P7064 except at the very late stage of infection (Fig. 4b). In parallel, we analyzed the expression of $A v r 1 b(+)$ and there was nearly no $A v r 1 b(+)$ transcripts detected in the virulent strain P6497, consistent with the results described previously (Shan et al., 2004). Both the avirulent allele $A v r 1 b^{\mathrm{P} 7064}$ and the virulent allele Avr $1 b^{\mathrm{P} 7076}$ were detected during infection (Fig. 4b).

We conclude that the $A v r 1 b$ locus is bidirectionally transcribed, leading to the development of dsRNA

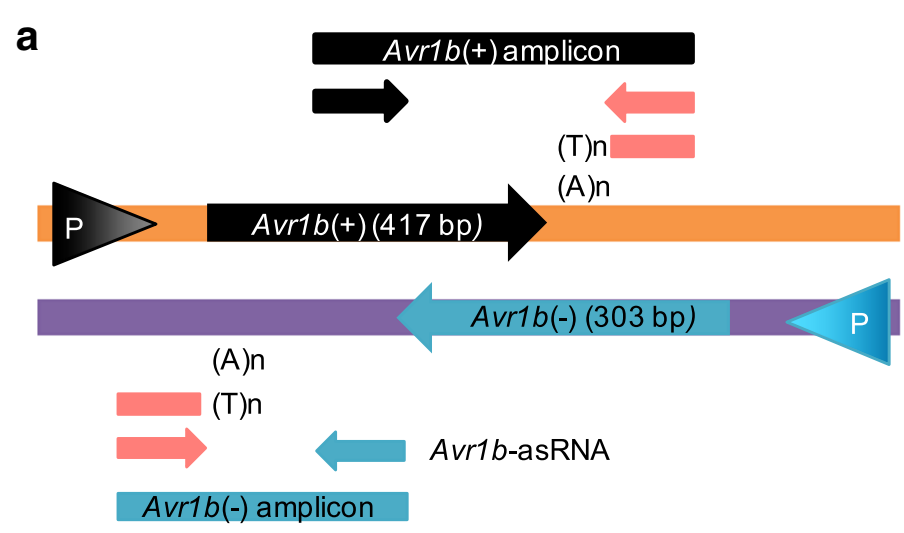

b

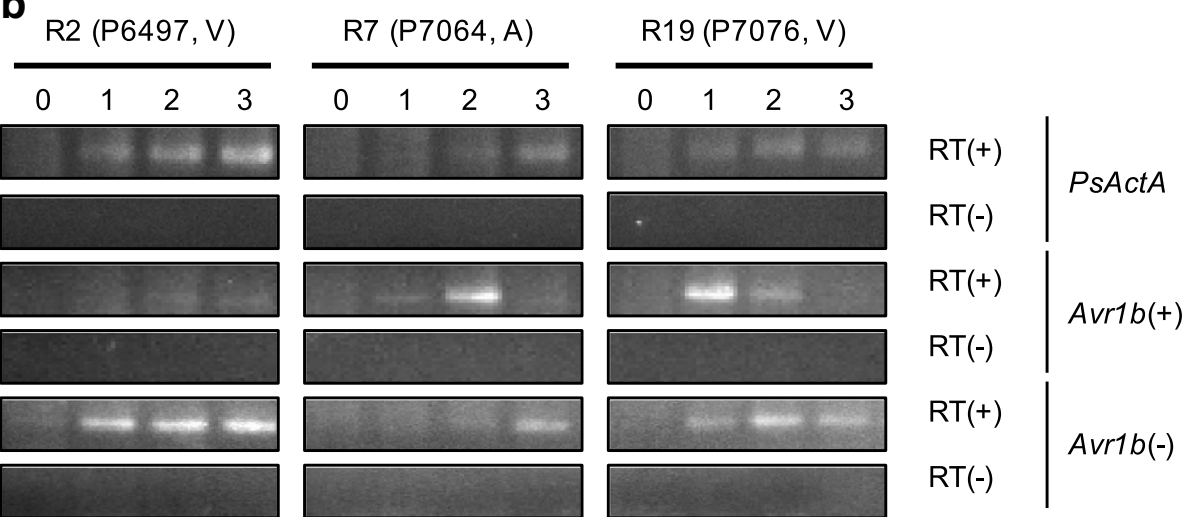

Fig. 4 Bidirectional transcription of $P$. sojae Avr1b locus. a The model and method for detection of the bidirectional transcription of the Avr1b locus. Avr $1 b(+)$ and $A v r 1 b(-)$ are organized in a tail-to-tail manner with more than 200 bp overlapping sequence. Oligo dT with an adaptor sequence (in red) was used for the first strand CDNA synthesis, and PCR was performed by using the combinations of Avr1b-asRNA sense (small blue arrow) primer, Avrib-asRNA antisense (small black arrow) and the adaptor (small red arrow) primers. P, the promoter. $\mathbf{b}$ RT-PCR validation of the bidirectional transcription. G. max cultivar Williams (rps 1b) infected with different strains of $P$. sojae were sampled at 0, 1, 2, and 3 dpi, respectively. Equal amount of total RNAs were used for each RT-PCR assays. RT(+) indicates the amplicon of RT-PCR, and RT(-) indicates the control reactions without cDNA synthesis (no reverse transcriptase applied). PsActA was amplified for 30 cycles and was used as a reference gene, while Avr1b(+) and Avr1b(-) were amplified for 35 cycles 
which may be the precursor of $A v r 1 b$-asRNA. Differential expression of $A v r 1 b(-)$ is responsible for differential accumulation of $A v r 1 b$-asRNA in P. sojae (Fig. $2 \mathrm{~b}$ and Fig. 4b). High level of $A v r 1 b$-asRNA ${ }^{\text {P6497 }}$ accumulation leads to the efficient degradation of $A v r 1 b^{\mathrm{P} 6497}$ transcripts and thus escaped from being detected (Fig. 4b), and $A v r 1 b$-siRNAs ${ }^{\mathrm{P} 6497}$ are the silencing signal molecules.

\section{Expression of large number of RXLR effector genes is associated with sRNAs}

To examine whether sRNA-associated silencing of RXLR effector genes was limited to the Avr effector genes, we mapped the deep sequencing sRNAs to the entire RXLR effector genes in $P$. sojae. In total, 125 RXLR effector genes (about 31\%) were found to be associated with the sRNA reads in strain P6497. Of these RXLR effector genes, 14 to 32 have comparable (or higher) sRNA levels than that of Avr1b sRNAs in P6497 (Fig. 5a, b).

To investigate if RXLR effector genes homologous sRNAs are associated with their silencing, we compared the expression levels of sRNA and the homologous RXLR effector genes. The results showed that the expressions of RXLR effector genes were negatively correlated with the number of sRNA reads (Fig. 5c). In particular, when the homologous sRNAs were highly expressed, nearly no transcripts of RXLR genes were detected (Fig. 5c).
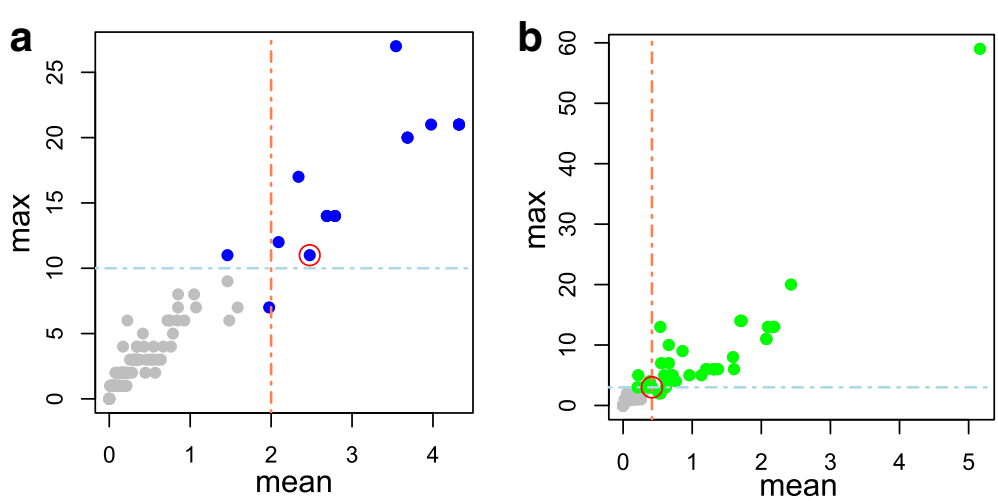

C

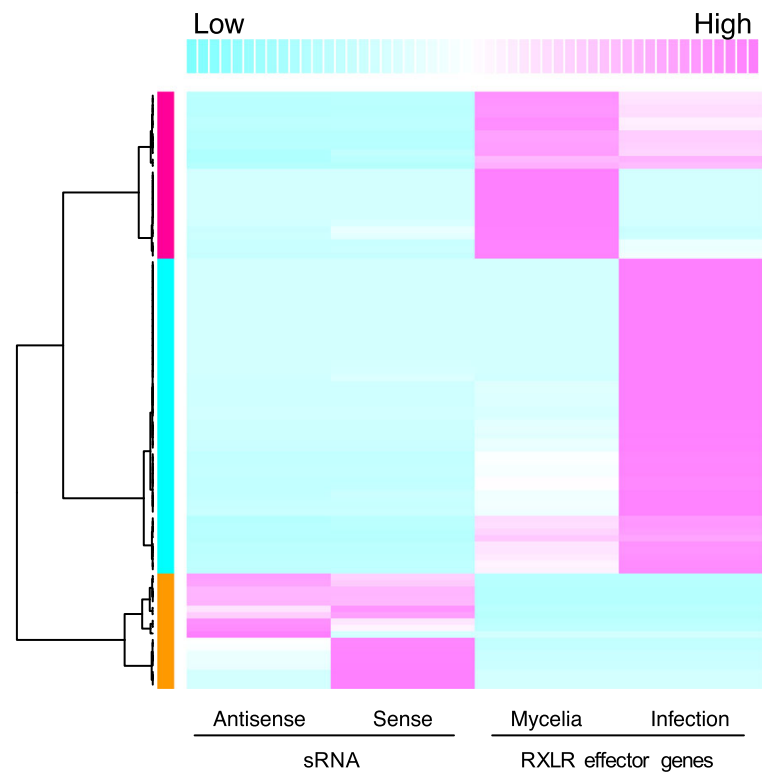

Fig. 5 The expression of numerous RXLR effector genes was negatively associated with the accumulation of homologous sRNAs in P. sojae. Screening of the high confident sRNA-associated RXLR effector genes by sense sRNA level (a) and antisense sRNA level (b). In $\mathbf{a}$ and $\mathbf{b}$, RXLR effector genes having comparable (or more) sRNA reads than the mean (x-axis) or max (y-axis) number of Avrib sRNA reads homologous to the ORF region were regarded as high confident sRNA associated. Blue or green dots indicate the RXLR effector genes above the threshold. Dashed lines indicate the threshold levels for the mean or max number of reads, and the red circle indicates Avr1b. c The sRNA expression was associated with silencing of RXLR effector genes in P. sojae in mycelia and during infection. The average abundance of sense and antisense sRNA reads of the RXLR effector genes, and the expression level of the RXLR effector genes in mycelia and infection tissues ( $24 \mathrm{~h}$ post inoculation) which retrieved from Phytophthora transcriptome database were used for clustering analysis 
Sequence variation in the regulatory regions is associated with bidirectional transcription of RXLR effector genes $A v r 1 b$ transcripts were not accumulated in virulent strain P6497, nor its antisense transcripts in the avirulent strain P7064. To examine underlying mechanism for the loss of antisense transcription of $A v r 1 b$ in strain P7064, we compared the Avr1b flanking sequences in strains P6497, P7064, P7074 and P7076 that represent four major genotypes of $P$. sojae (Fig. 6a). The major difference within the 500-bp flanking sequences among these four strains is a 10-bp deletion in the antisense Avr1b promoter region in P7064 (deletion a, Fig. 6a). Interestingly, we also identified a 10-bp deletion in the promoter region of $A v r 1 b$ in the virulent strain P7074 (deletion b, Fig. 6a), which has no (or much less) accumulation of $A v r 1 b$ transcripts (Shan et al. 2004).

The two independent deletions detected in the regulatory regions suggest that these 10 -bp deletions may play an important cis-regulatory role in the expression of $A v r 1 b$. This further suggests possible wide-spread presence of these 10-bp deletions in the gene regulatory regions across the genome of $P$. sojae. We hence performed genome-wide identification for the INDEL (insertion and deletion) variations in all the gene regulatory regions of $P$. sojae genes. By comparison of those INDEL variants identified in the three re-sequenced $P$. sojae genomes (Wang et al. 2011), we found that most of the INDELs in the promoters are $1 \mathrm{bp}$ in length, and the frequency declines as the INDEL length increases (Fig. 6b). In contrast, the frequency of 9-bp and 10-bp INDEL variants were significantly increased (Fig. 6b, in red), especially in the RXLR effector genes (Fig. 6c). This suggests that the 9-bp and 10-bp INDEL variants might have undergone evolutionary selection and function as cis-elements that regulate gene expression.

\section{Discussion}

Virulence variation is critical for pathogen survival and is responsible for loss of disease resistance in a wide range of host plants against diverse pathogens. In this study, we showed that virulence variation of $P$. sojae is associated with accumulation of sRNAs homologous to

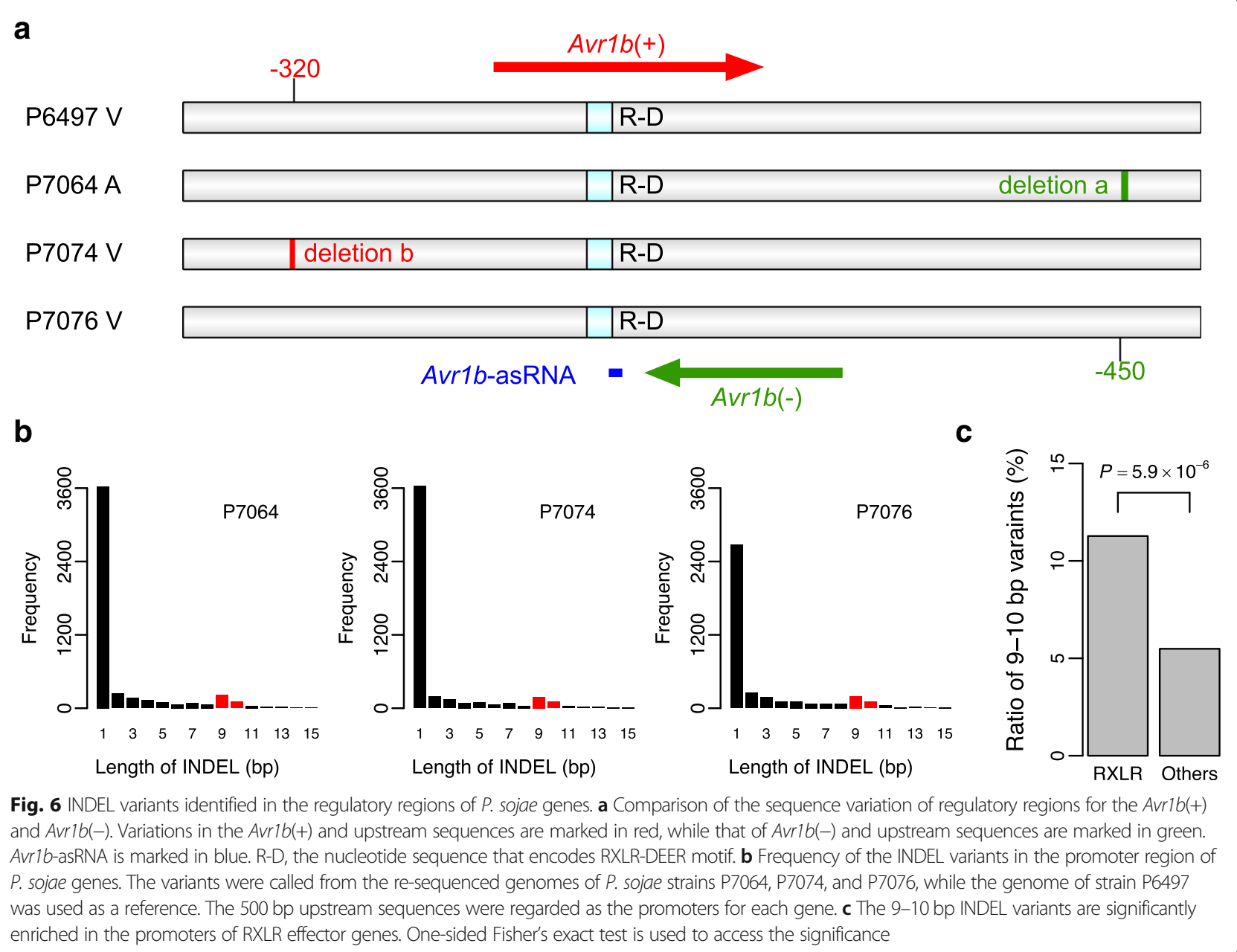


avirulence genes. While the complete $A v r 1 b$ gene sequences are present in P. sojae strains P6497 and P7064, but no Avr1b mRNA accumulation was detected during infection by P6497 (Shan et al. 2004). The Avr1b-asRNA is derived from the antisense of Avr1b, which has distinct expression patterns in strains P6497 and P7064. In the avirulent P7064, the antisense of $A v r 1 b$ was not detectable in the early stage of infection. Further analysis revealed that this was likely due to a 10-bp deletion in the promoter region of $\operatorname{Avr} 1 b(-)$.

We propose a sRNA-based model (Fig. 7) to explain the results presented in this study. During the early stage of plant infection, Avr1 $b^{\mathrm{P} 6497}$ transcript and its antisense transcript formed NATs, which is recognized by endogenous RNase and processed to form Avr1b-asRNA. This sRNA could lead Avr1b silencing in strain P6497 at posttranscriptional level. In the avirulent strain P7064, the lack of Avr1b(-) ${ }^{\mathrm{P} 7064}$ leads to a failure of dsRNA formation, and therefore is unable to produce the corresponding sRNA and the silencing signals in the avirulent strain. A similar model has been proposed in Arabidopsis infected by bacterial pathogen Pseudomonas syringae carrying effector avrRpt2, in which the silencing of PPRL gene was caused by nat-siRNAATGB2, a sRNA derived from the NATs of $A T G B 2$ and PPRL in Arabidopsis (Katiyar-Agarwal et al. 2006). In Arabidopsis, the biogenesis of siRNAATGB2 requires DCL1, RDR6, HEN1, SGS3, HYL3, and Pol IVa (Katiyar-Agarwal et al. 2006). It is quite possible that their homologs in $P$. sojae are also involved in the biogenesis of Avr1b-asRNA.
We have generated several lines of evidence that support our hypothesis (Fig. 7). Firstly, P. sojae encodes sRNAs that are associated with Avrlb silencing and virulence variation. By deep sequencing data analysis, we discovered two species of sRNAs: the trigger and silencing signal sRNA molecules. The Avrlb-asRNA is about $40 \mathrm{nt}$ in size, different from the miRNAs and siRNAs identified in oomycetes (Vetukuri et al. 2012; Fahlgren et al. 2013; Jia et al. 2017). Although most oomycete sRNAs are $21 \mathrm{nt}$ or 25/26 nt in size, larger sRNAs are also frequently observed in P. sojae and P. infestans (Vetukuri et al. 2011b; Vetukuri et al. 2012; Wang et al. 2016), and Arabidopsis (Katiyar-Agarwal et al. 2007). The siRNAs are 25-26 nt in oomycete and are recognized as the signals of gene silencing (Vetukuri et al. 2012; Qutob et al. 2013; Jia et al. 2017). Consistently, the Avr1b-siRNAs are $25 \mathrm{nt}$ and associated with the virulence variation in $P$. sojae. In addition, we showed that a large number of sRNAs are associated with the RXLR effector gene silencing in $P$. sojae, suggesting that sRNA may have been associated broadly with expression regulation of RXLR effector genes. In Phytophthora, it is also shown that many sRNAs are homologous to RXLR and CRN effector genes (Vetukuri et al. 2012; Jia et al. 2017). Based on the accumulation and mapping patterns, we conclude that $A v r 1 b$-asRNA is likely the trigger sRNA and is responsible for the production of the signal siRNAs.

Secondly, bidirectional transcription and dosage compensation are simultaneously involved in the silencing of $A v r 1 b$ gene. We showed that $A v r 1 b$-asRNA is derived from

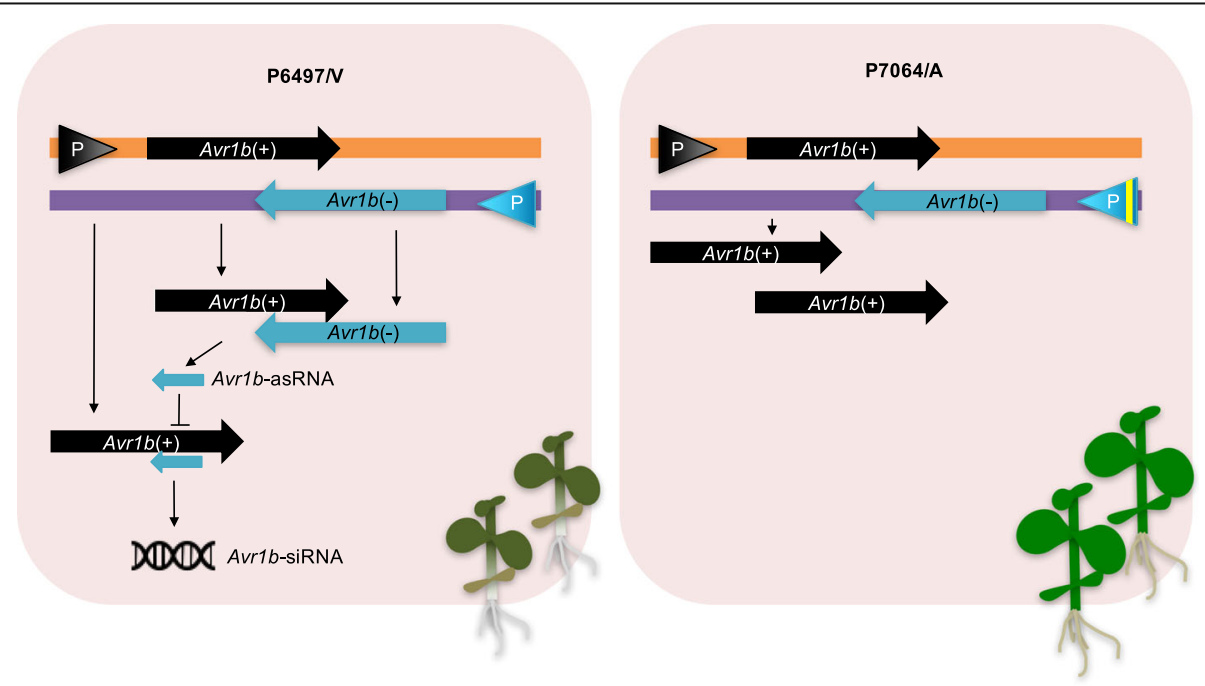

Fig. 7 A proposed molecular model for the regulation of Avrlb expression in the avirulent and virulent $P$. sojae strains. Both P. sojae strains P6497 and P7064 encodes Avrib(+)and Avrlb(-) genes. In the virulent strain P6497 (the left panel), Avrib(+) ${ }^{\mathrm{P} 6497}$ and Avrib(-) ${ }^{\mathrm{P} 6497}$ are transcribed in a bidirectional manner and formed NATs during infection; the NATs are recognized by the putative endogenous cleavage machinery to produce Avrlb-asRNA ${ }^{\mathrm{P} 6497}$. The produced sRNA targets on the newly transcribed Avr1b(+) ${ }^{\mathrm{P} 649}$ and generates abundant Avr1b-siRNA ${ }^{\mathrm{P} 6497}$ that function as the RNA silencing signals, leading to abolished $A v r 1 b(+)^{\mathrm{P} 6497}$ function and escaped Rps 16 recognition. In the avirulent strain P7064 (the right panel), a 10-bp deletion in Avrlb(-) ${ }^{\mathrm{P} 7064}$ promoter region leads to significantly reduced $A v r 1 b(-)^{\mathrm{P} 7064}$ transcription and a failure of $A v r 1 b(+)^{\mathrm{P} 7064}$ silencing, allowing Avr1b(+) ${ }^{\mathrm{P} 7064}$ be recognized by Rps1b 
$A v r 1 b(-)$, and thus discovered that the Avr1b locus is transcribed bidirectionally, as revealed by sequence and strand-specific RT-PCR analyses. The NATs formed by the bidirectional transcripts from Avr $1 b$ locus were the precursor of $A v r 1 b$-asRNA. NATs are commonly present and have regulatory roles in eukaryotes (Jen et al. 2005; Wang et al. 2005; Katiyar-Agarwal et al. 2006). In P. sojae, our preliminary investigation revealed that about 400 loci, including RXLR effector genes, have the potential to form NATs. Avr1b(-) ${ }^{\mathrm{P} 6497}$ and $A v r 1 b(-)^{\mathrm{P} 7076}$ were abundantly expressed, being positively correlated with the supressed expression of $A v r 1 b(+)^{\mathrm{P} 6497}$ and $A v r 1 b(+)^{\mathrm{P} 7076}$. Furthermore, the expression level of $A v r 1 b(-)^{\mathrm{P} 6497}$ was much higher than Avr1b(-) ${ }^{\mathrm{P} 7076}$, being consistent with the fact that $A v r 1 b(+)^{\mathrm{P} 6497}$ has no accumulation while $A v r 1 b(+)^{\mathrm{P} 7076}$ is still detectable. Consistently, Avrlb-asR$\mathrm{NA}^{\mathrm{P} 6497}$ was much higher than Avr1b-asRNA ${ }^{\mathrm{P} 7076}$. Therefore, it is likely that there is an expression threshold for $A v r 1 b(-)$ to generate sufficient sRNA to trigger silencing of Avr1b(+).

Finally, expression of Avr1b sense and antisense transcripts are likely programmed by the INDEL variations in their promoter sequences. Our analysis of the flanking sequences revealed that the differential Avr $1 b(+/-)$ expression was associated with the INDEL variations of promoter sequences. By comparing the sequence of strains P7074 and P7064, we found that the loss of $A v r 1 b(+)^{\mathrm{P} 7074}$ expression is likely associated with a 10-base deletion in $\operatorname{Avr} 1 b(+)^{\mathrm{P} 7074}$ promoter. By comparing the sequence of strains P7064 and P6497, the loss of $A v r 1 b(-)^{\mathrm{P} 7064}$ expression is likely associated with a 10-bp deletion in $\operatorname{Avr} 1 b(-)^{\mathrm{P} 7064}$ promoter. A further genome-wide analysis suggests that 9 or 10 -bp INDEL variations were broadly present in the promoter regions of $P$. sojae genes and were significantly enriched in the RXLR effector genes, suggesting that the oomycete pathogens may have evolved functional cis-elements by promoter INDEL variations to control gene expression, especially for the RXLR effector genes. Consistently, a recent study showed that a natural 10-bp INDEL variant of the $P A X 7$ promoter could regulate gene expression by altering the binding of transcriptional factor ZNF219 in animal cells (Xu et al. 2018). Notably, $10 \mathrm{bp}$ is the distance of the double helix per turn in DNA, allowing the distance of cis-elements be easily reprogrammed by INDEL variations. Since the variation of expression of RXLR effector genes is essential for the virulence variation, these INDEL in the promoter regions may be important in shaping the plasticity of the expressions of RXLR effector genes.

\section{Methods}

\section{Phytophthora sojae strains and culture conditions}

P. sojae strains P6497 (Race 2, Avr1b ${ }^{-}$), P7064 (Race 7, $A v r 1 b^{+}$), P7074 (Race 17, Avr1b $b^{-}$) and P7076 (Race 19, $A v r 1 b^{-}$) representing four major genotypes and nearly all the genetic variations (Forster et al. 1994) were used in this study. P. sojae cultures were routinely maintained on $5 \%(\mathrm{v} / \mathrm{v})$ carrot juice agar (CA) medium supplemented with $0.002 \%(w / v) ~ \beta$-sitosterol and $0.01 \%(w / v) \mathrm{CaCO}_{3}$, and incubated at $25^{\circ} \mathrm{C}$ in the dark.

\section{Soybean inoculation procedure}

Soybean (Glycine max) cultivars Williams (rps1b) and Williams L77-1863 (Rps1b) were used for P. sojae infection assay. The 8-day-old seedlings were wound inoculated at hypocotyls with a vertical slit of about $1 \mathrm{~cm}$ in length, and a small piece of $P$. sojae mycelia were then mounted at the wound. The hypocotyls were cut from the upper $0.5 \mathrm{~cm}$ to the lower $0.5 \mathrm{~cm}$ (total $2 \mathrm{~cm}$ including the wound) 0 to 3 days post inoculation (dpi). Williams infected tissues were used for Northern blot and RT-PCR analyses, while Williams L77-1863 carrying Rps1b resistance gene was used as a control to monitor the phenotype.

\section{Phytophthora sojae and soybean RNA preparation}

RNA was isolated by using TRIzol (Invitrogen, USA). In brief, the infected hypocotyl tissues were ground into fine powder with liquid nitrogen, treated with TRIzol at room temperature, followed by extraction with chloroform and phenol-chloroform. RNA precipitation was performed by isopropyl alcohol overnight at $-20^{\circ} \mathrm{C}$ to fully recover sRNAs. Finally, the sRNA pellets were washed with ethanol and were dissolved with DEPC-treated $\mathrm{ddH}_{2} \mathrm{O}$.

\section{Northern assay for sRNA and siRNA detections}

Northern analysis was performed as described previously (Wang et al. 2016). In brief, $20 \mu \mathrm{g}$ of total RNAs were denatured and subjected to a 15\% urea-denaturing PAGE. RNAs were transferred onto nylon membranes and were fixed by a UV-crosslinker (HL-2000 HybriLinker, UVP) followed by baking at $80^{\circ} \mathrm{C}$ for $2 \mathrm{~h}$. Pre-hybridization and hybridization were carried out at $42^{\circ} \mathrm{C}$ for $8 \mathrm{~h}$, respectively, in a Hybridizer (HL-2000 HybriLinker, UVP). The membranes were washed twice by $2 \times \mathrm{SSC}$ and $0.5 \%$ SDS at $37^{\circ} \mathrm{C}$ for $15 \mathrm{~min}$. The images were exposed and detected by the FLA-7000 image system (Fujifilm, Japan).

For Avr1b-asRNA detection, DNA oligonucleotides (Avr1b-asRNA-probe: 5' -TCA GTC ACG CTG AAG GTC CGC TCC C-3'), complementary to the Avr1b-asRNA, was labeled with $\left[\gamma^{-32} \mathrm{P}\right]$-ATP using T4 Polynucleotide Kinase (TaKaRa, China). For Avrlb-siRNAs detection, the ORF sequences were amplified from the genomic DNA and labeled with $\left[\alpha-{ }^{32} \mathrm{P}\right]-\mathrm{dCTP}$ using Random Primer DNA Labeling Kit (TaKaRa, China).

\section{RT-PCR analysis}

RT-PCR assay was performed to verify gene transcription. To confirm the expression of Avr1b and its antisense transcript, the first strand cDNA was synthesized 
using $1 \mu \mathrm{g}$ total RNA with oligo dT carrying an adaptor (OldTail: 5'-GAG CAG TCG AGA CTC GAT CGC ATG CAT TTT TTT TTT TTT TTT TTV N-3') by using Reverse Transcriptase M-MLV (TaKaRa, China). Two gene-specific primers (GSP) designed according to the $A v r 1 b$ and its antisense sequences (GSP+: 5' -GGG AGC GGA CCT TCA GCG TGA CTG A-3'; GSP-: 5'-TCA GTC ACG CTG AAG GTC CGC TCC C-3') and the adapter primer (Tail: 5'-GCA GTC GAG ACT CGA TCG CAT-3') were used as the forward and reverse primers, respectively.

\section{Bioinformatics analysis}

For the identification of $A v r 1 b$-asRNA and $A v r 1 b$-siRNA, the sRNA reads (SRR4450568) (Wang et al. 2016) were mapped to the open reading frame (ORF) of $A v r 1 b^{\mathrm{P} 6497}$ by using bowtie (Langmead et al. 2009) with no mismatch allowed as described previously. Mapping of the reads to the entire RXLR effector genes was done as that of Avr1b. The expression data of RXLR effector genes were retrieved from Phytophthora transcriptome database (Ye et al. 2011).

For the analysis of Avrlb regulatory sequences, $A v r 1 b^{\mathrm{P} 6497}$ and its flanking sequences were retrieved from P. sojae genome (P6497, version 3) (Tyler et al. 2006) and were used for identification of the contigs in P. sojae strains P7064, P7074, and P7076 (Wang et al. 2011). The flanking sequences of P7064 and P7074 were derived from single contigs (contig29872 and contig03312, respectively) and P7076 from two separate contigs (contig01119 and contig02466) in the 454 assemblies. The retrieved sequences were confirmed by sequencing at GenScript (Nanjing, China). Multiple sequence alignment was performed with Clustal X2 (Larkin et al. 2007) and visualized via DOG (Ren et al. 2009) The promoter sequences were predicted by EP3 (Abeel et al. 2008).

The identification of INDEL variants was performed as described previously (Wang et al. 2017). Briefly, the contigs of the re-sequenced $P$. sojae genomes of strains P7064, P7074, and P7076 (Wang et al. 2011) were re-ordered by ABACAS (Assefa et al. 2009) with the reference genome of strain P6497 (Tyler et al. 2006), and MUMmer (Delcher et al. 1999) was used to call the INDEL variants with its default parameters. According to previous study, the 5' UTR regions are very short (Win et al. 2006), thus the 500-bp upstream sequences were regarded as the promoters for each gene.

\section{Abbreviations}

asRNA: antisense small RNA; CRN: Crinkler; INDEL: Insertion and deletion; NAT: Natural antisense transcript; ORF: Open reading frame; RT-PCR: Reverse transcription polymerase chain reaction; RXLR: Arg-Xaa-Leu-Arg, Xaa could be any amino acid; siRNA: small interfering RNA; sRNA: small RNA;

UTR: Untranslated region

\section{Acknowledgements}

We thank Drs Ming-Bo Wang and Meixiang Zhang for helpful and fruitful discussions.

\section{Funding}

This work was supported by China Agriculture Research System (CARS-09), National Natural Science Foundation of China (31701747 and 31561143007 ), the Programme of Introducing Talents of Innovative Discipline to Universities (Project 111) from the State Administration of Foreign Experts Affairs (\#B18042) and China Postdoctoral Science Foundation (2015M580884, and 2017M623262).

\section{Availability of data and materials}

All data generated or analyzed during this study are included in this published article.

\section{Authors' contributions}

WS and QW conceived the research; QW, TL, CZ, SL, KX, and YM performed the experiment; $\mathrm{QW}$ and WS performed data analysis; $\mathrm{QW}, \mathrm{BG}, \mathrm{BMT}$, and WS wrote the paper with contributions from all authors. All authors read and approved the final manuscript.

Ethics approval and consent to participate

Not applicable.

\section{Consent for publication}

Not applicable.

\section{Competing interests}

The authors declare that they have no competing interests.

\section{Author details}

${ }^{1}$ State Key Laboratory of Crop Stress Biology for Arid Areas, and College of Plant Protection, Northwest A\&F University, Yangling 712100, Shaanxi, China. ${ }^{2}$ State Key Laboratory of Crop Stress Biology for Arid Areas, and College of Agronomy, Northwest A\&F University, Yangling 712100, Shaanxi, China.

${ }^{3}$ Center for Genome Research and Biocomputing and Department of Botany and Plant Pathology, Oregon State University, Corvallis, Oregon 97331, USA.

Received: 25 January 2019 Accepted: 7 May 2019

Published online: 24 May 2019

\section{References}

Abeel T, Saeys Y, Bonnet E, Rouze P, Van de Peer Y. Generic eukaryotic core promoter prediction using structural features of DNA. Genome Res. 2008;18:310-23.

Abu-El Samen FM, Secor GA, Gudmestad NC. Variability in virulence among asexual progenies of Phytophthora infestans. Phytopathology. 2003;93:293-304.

Anderson RG, Deb D, Fedkenheuer K, McDowell JM. Recent progress in RXLR effector research. Mol Plant-Microbe Interact. 2015;28:1063-72.

Assefa S, Keane TM, Otto TD, Newbold C, Berriman M. ABACAS: algorithm-based automatic contiguation of assembled sequences. Bioinformatics. 2009;25:1968-9.

Delcher AL, Kasif S, Fleischmann RD, Peterson J, White O, Salzberg SL. Alignment of whole genomes. Nucleic Acids Res. 1999:27:2369-76.

Fahlgren N, Bollmann SR, Kasschau KD, Cuperus JT, Press CM, Sullivan CM, et al. Phytophthora have distinct endogenous small RNA populations that include short interfering and microRNAs. PLoS One. 2013;8:e77181.

Forster H, Tyler BM, Coffey MD. Phytophthora sojae races have arisen by clonal evolution and by rare outcrosses. Mol Plant-Microbe Interact. 1994;7:780-91.

Haas BJ, Kamoun S, Zody MC, Jiang RHY, Handsaker RE, Cano LM, et al. Genome sequence and analysis of the Irish potato famine pathogen Phytophthora infestans. Nature. 2009;461:393-8.

Haverkort AJ, Boonekamp PM, Hutten R, Jacobsen E, Lotz LAP, Kessel GJT, et al. Societal costs of late blight in potato and prospects of durable resistance through cisgenicmodification. Potato Res. 2008;51:47-57.

Jen $\mathrm{CH}$, Michalopoulos I, Westhead DR, Meyer P. Natural antisense transcripts with coding capacity in Arabidopsis may have a regulatory role that is not linked to double-stranded RNA degradation. Genome Biol. 2005;6:R51.

Jia J, Lu W, Zhong C, Zhou R, Xu J, Liu W, et al. The 25-26 nt small RNAs in Phytophthora parasitica are associated with efficient silencing of homologous endogenous genes. Front Microbiol. 2017:8:773.

Jiang RH, Tyler BM. Mechanisms and evolution of virulence in oomycetes. Annu Rev Phytopathol. 2012;50:295-318 
Jiang RHY, Tripathy S, Govers F, Tyler BM. RXLR effector reservoir in two Phytophthora species is dominated by a single rapidly evolving superfamily with more than 700 members. Proc Natl Acad Sci U S A. 2008;105:4874-9. Jones JDG, Dangl JL. The plant immune system. Nature. 2006;444:323-9.

Judelson HS, Tani S. Transgene-induced silencing of the zoosporogenesis-specific NIFC gene cluster of Phytophthora infestans involves chromatin alterations. Eukaryot Cell. 2007;6:1200-9.

Kamoun S. A catalogue of the effector secretome of plant pathogenic oomycetes. Annu Rev Phytopathol. 2006;44:41-60.

Kamoun S, Furzer O, Jones JD, Judelson HS, Ali GS, Dalio R, et al. The top 10 oomycete pathogens in molecular plant pathology. Mol Plant Pathol. 2015;16:413-34.

Katiyar-Agarwal S, Gao S, Vivian-Smith A, Jin H. A novel class of bacteria-induced small RNAs in Arabidopsis. Genes Dev. 2007;21:3123-34.

Katiyar-Agarwal S, Morgan R, Dahlbeck D, Borsani O, Villegas A, Zhu JK, et al. A pathogen-inducible endogenous siRNA in plant immunity. Proc Natl Acad Sci U S A. 2006;103:18002-7

Langmead B, Trapnell C, Pop M, Salzberg SL. Ultrafast and memory-efficient alignment of short DNA sequences to the human genome. Genome Biol. 2009;10:R25.

Larkin MA, Blackshields G, Brown NP, Chenna R, McGettigan PA, McWilliam H, et al. Clustal W and Clustal X version 2.0. Bioinformatics. 2007;23:2947-8.

Malvick DK, Percich JA. Variation in pathogenicity and genotype among singlezoospore strains of Aphanomyces euteiches. Phytopathology. 1998;88:52-7.

Oh SK, Young C, Lee M, Oliva R, Bozkurt TO, Cano LM, et al. In planta expression screens of Phytophthora infestans RXLR effectors reveal diverse phenotypes, including activation of the Solanum bulbocastanum disease resistance protein Rpi-blb2. Plant Cell. 2009;21:2928-47.

Qutob D, Chapman BP, Gijzen M. Transgenerational gene silencing causes gain of virulence in a plant pathogen. Nat Commun. 2013;4:1349.

Qutob D, Tedman-Jones J, Dong S, Kuflu K, Pham H, Wang Y, et al. Copy number variation and transcriptional polymorphisms of Phytophthora sojae RXLR effector genes Avrla and Avr3a. PLoS One. 2009;4:e5066.

Ren J, Wen L, Gao X, Jin C, Xue Y, Yao X. DOG 1.0: illustrator of protein domain structures. Cell Res. 2009;19:271-3.

Rutherford FS, Ward EWB, Buzzell RI. Variation in virulence in successive singlezoospore propagations of Phytophthora megasperma f. sp. glycinea. Phytopathology. 1985;75:371-4.

Shan WX, Cao M, Dan LU, Tyler BM. The Avr1b locus of Phytophthora sojae encodes an elicitor and a regulator required for avirulence on soybean plants carrying resistance gene Rps 16. Mol Plant-Microbe Interact. 2004;17:394-403.

Song T, Kale SD, Arredondo FD, Shen D, Su L, Liu L, et al. Two RxLR avirulence genes in Phytophthora sojae determine soybean Rps1k-mediated disease resistance. Mol Plant-Microbe Interact. 2013;26:711-20.

Stassen JHM, Van den Ackerveken G. How do oomycete effectors interfere with plant life? Curr Opin Plant Biol. 2011;14:407-14

Tyler BM. Phytophthora sojae: root rot pathogen of soybean and model oomycete. Mol Plant Pathol. 2007:8:1-8.

Tyler BM, Tripathy S, Zhang X, Dehal P, Jiang RH, Aerts A, et al. Phytophthora genome sequences uncover evolutionary origins and mechanisms of pathogenesis. Science. 2006;313:1261-6.

van West P, Kamoun S, van 't Klooster JW, Govers F. Internuclear gene silencing in Phytophthora infestans. Mol Cell. 1999;3:339-48.

van West P, Shepherd SJ, Walker CA, Li S, Appiah AA, Grenville-Briggs LJ, et al. Internuclear gene silencing in Phytophthora infestans is established through chromatin remodelling. Microbiology. 2008;154:1482-90.

Vetukuri RR, Asman AK, Tellgren-Roth C, Jahan SN, Reimegard J, Fogelqvist J, et al. Evidence for small RNAs homologous to effector-encoding genes and transposable elements in the oomycete Phytophthora infestans. PLoS One. 2012;7:e51399.

Vetukuri RR, Avrova AO, Grenville-Briggs $\sqcup$, Van West P, Soderbom F, Savenkov El, et al. Evidence for involvement of dicer-like, Argonaute and histone deacetylase proteins in gene silencing in Phytophthora infestans. Mol Plant Pathol. 2011a;12:772-85.

Vetukuri RR, Tian ZD, Avrova AO, Savenkov El, Dixelius C, Whisson SC. Silencing of the PiAtvr3a effector-encoding gene from Phytophthora infestans by transcriptional fusion to a short interspersed element. Fungal Biol. 2011b;115:1225-33.

Wang Q, Jiang C, Wang C, Chen C, Xu JR, Liu H. Characterization of the two-speed subgenomes of Fusarium graminearum reveals the fast-speed subgenome specialized for adaption and infection. Front Plant Sci. 2017;8:140

Wang Q, Li T, Xu K, Zhang W, Wang X, Quan J, et al. The tRNA-derived small RNAs regulate gene expression through triggering sequence-specific degradation of target transcripts in the oomycete pathogen Phytophthora sojae. Front Plant Sci. 2016;7:1938.
Wang QQ, Han CZ, Ferreira AO, Yu XL, Ye WW, Tripathy S, et al. Transcriptional programming and functional interactions within the Phytophthora sojae RXLR effector repertoire. Plant Cell. 2011;23:2064-86.

Wang XJ, Gaasterland T, Chua NH. Genome-wide prediction and identification of cisnatural antisense transcripts in Arabidopsis thaliana. Genome Biol. 2005;6:R30.

Win J, Kanneganti TD, Torto-Alalibo T, Kamoun S. Computational and comparative analyses of 150 full-length cDNA sequences from the oomycete plant pathogen Phytophthora infestans. Fungal Genet Biol. 2006;43:20-33.

Wrather JA, Koenning SR. Estimates of disease effects on soybean yields in the United States 2003 to 2005. J Nematol. 2006;38:173-80.

Xu Y, Shi T, Zhou Y, Liu M, Klaus S, Lan X, et al. A novel PAX7 10-bp indel variant modulates promoter activity, gene expression and contributes to different phenotypes of Chinese cattle. Sci Rep. 2018;8:1724.

Ye W, Wang X, Tao K, Lu Y, Dai T, Dong S, et al. Digital gene expression profiling of the Phytophthora sojae transcriptome. Mol Plant-Microbe Interact. 2011:24:1530-9.
Ready to submit your research? Choose BMC and benefit from:

- fast, convenient online submission

- thorough peer review by experienced researchers in your field

- rapid publication on acceptance

- support for research data, including large and complex data types

- gold Open Access which fosters wider collaboration and increased citations

- maximum visibility for your research: over $100 \mathrm{M}$ website views per year

At $\mathrm{BMC}$, research is always in progress.

Learn more biomedcentral.com/submissions 